\title{
Adaptive bitrate and resource allocation for relay-assisted ARQ transmissions
}

\author{
Adrian Agustin, Member IEEE, Josep Vidal, Member IEEE and Olga Muñoz
}

\begin{abstract}
Automatic repeat request (ARQ) protocols deal with the situations where the selected data rate is not supported by the current channel realization. The inappropriate selection is motivated by the limited channel state information at the transmitter (CSIT). This work looks into how the ARQ protocols are applied to the half-duplex relay-assisted transmission with decode-and-forward relays by exploiting the ACK feedback channel of the terminals. Two implementations are tackled: 1) only the source or 2) source and relay manage the retransmissions under the pure-ARQ protocol. This work presents an analytical framework for the comparison of both options. The study allows the optimization of the bitrate and amount of resource allocated for the relay transmission phases.
\end{abstract}

\section{Index Terms - ARQ, relay, cooperative system}

\section{INTRODUCTION}

Relay-assisted has become an effective tool for combating the effects of propagation channel in wireless networks thanks to the relay terminal. Originally proposed in [1] with capacity results proved in [2], it has regained attention due to the diversity gain obtained in wireless channels, [3],[4],[5]. This work assumes half-duplex relays under decode-and-forward (DF), [4]. Hence, the transmission is carried out through two orthogonal phases: relay-receive phase (or phase I) and relaytransmit phase (or phase II).

Among the many existing relay protocols [6], we have selected the relay-assisted transmission described by Fig. 1. In phase I the source transmits to the relay and destination. Afterwards, only the relay transmits to the destination. This is the same procedure used in [4] and named protocol I in [6]. The relay may transmit under repetition coding, $\mathrm{RC}$, (using the same codeword as the source) or unconstrained coding, UC, (employing a different codebook from the source), [8].

The exploitation of the ACK feedback channels from the terminals allows implementing the relay-assisted transmission procedure in different ways. For instance, if the source is in charge of the Automatic Repeat reQuest (ARQ) procedure and the source uses only the requests from destination, the retransmission is repeated along phase I and phase II. If the

This work was supported in part by the European Union through project ROCKET ICT-2007-1-215282 and FEDER funds, and by the Spanish/Catalan Science and Technology Commissions through projects: 2005SGR-00639, TEC2006-06481/TCM, TEC2004-04526 and CONSOLIDER CSD200800010 COMONSENS

A.Agustin, J.Vidal and O.Muñoz are with the Signal Theory and Communication Dept. at UPC, Barcelona, Spain. Email: \{adrian.agustin, josep.vidal, olga.munoz\}@upc.edu relay feedbacks to the source its success (ACK/NACK) in decoding the message, then the source might transmit during phase II, remaining the relay silent. Similarly, an ARQ protocol proposed in [9] enforces the use of the relay only when it receives successfully in phase $I$ of the first transmission, also with ACKs from relay and destination. That analysis is carried out for the pure- $A R Q$ protocol [10] with adaptive modulation. Moreover, the optimal bitrate and resource allocation is addressed in [7], where the source manages the ARQ with ACKs only from destination.

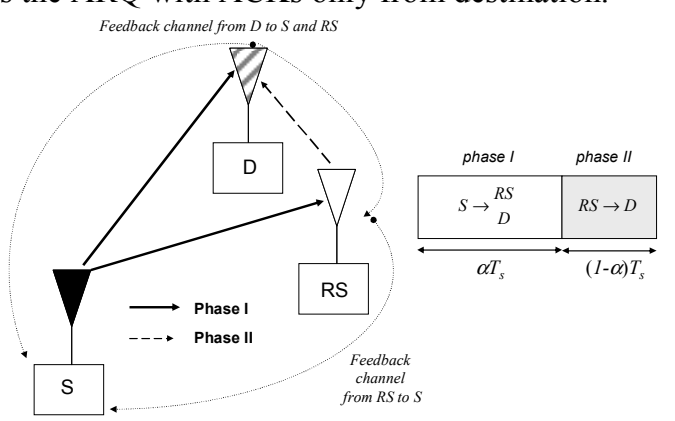

Fig. 1. Relay-assisted transmission. S(source), RS(relay) and D(destination)

When source and relay control the ARQ procedure, the source receives the feedback from the relay and destination while the relay listens the feedback from destination (ACKs from relay and destination). In such a case, the source transmits till either the relay or destination decodes the message. Once the relay has decoded, the source is freed and only the relay retransmits to the destination. When applied to the downlink of cellular systems the resources are employed more efficiently, since the source-to-destination link exhibits lower signal to noise ratio (SNR) than the relay-to-destination link. This ARQ protocol introduced in [11] follows the transmission depicted in Fig. 1 and it outlines the benefits of using different assisting relays in each retransmission through a numerical evaluation of the throughput for a given data rate. Likewise, the situation where source and relay transmits simultaneously in phase II is analyzed in [12] from a multiplexing-diversity-delay tradeoff point of view, using an incremental redundancy Hybrid ARQ.

The present work envisions the pure-ARQ, [10], when onlysource or source-and-relay manage the retransmission procedure, in contrast to [7]. Moreover, unlike [11] and [9], the transmission rate and times of phase I and phase II are optimized to maximize the throughput. The main contributions are:

- We show that the best throughput is obtained when source and relay manage the retransmissions. 
- We derive closed-form and integral expressions for throughput.

- We elucidate which positions for the relay terminal are the best to achieve the highest throughput.

\section{SCENARIO AND SIGNAL MODEL}

The scenario consists of a source, a half-duplex DF relay and a destination. All terminals are single-antenna. Source and relay transmit with constant power $P$ over time slots of duration $T_{s}$. For sake of notation simplicity the bandwidth allocated is $1 \mathrm{~Hz}$. Transmission time is much shorter than the coherence time of the channel. The channel is modeled by an independent and identically distributed (i.i.d.) process with a Rayleigh distribution across successive transmission rounds. The mutual information in bits at each link is given by,

$$
I_{i}=\log _{2}\left(1+\gamma_{i}\right) \text { bits / channel use }
$$

where $\gamma_{i}$ follows an exponential distribution with mean $\rho_{i}$,

$$
\rho_{0}=\frac{P}{L_{0} \sigma_{d}^{2}} \quad \rho_{1}=\frac{P}{L_{1} \sigma_{r}^{2}} \quad \rho_{2}=\frac{P}{L_{2} \sigma_{d}^{2}}
$$

where $i=0,1$ and 2 stand for the source-destination, sourcerelay and relay-destination links, $L_{\mathrm{i}}$ defines the pathloss and $\sigma_{d}^{2}$ and $\sigma_{r}^{2}$ are the noise power at destination and relay.

The relay-assisted transmission is divided in phase I and phase II of time duration $\alpha T_{s}$ and (1- $\left.\alpha\right) T_{s}$, respectively, see Fig.1. The relay transmits using repetition coding (RC) or with unconstrained coding (UC). We characterize the success of the transmission by the outage probability, i.e. the probability that the selected data rate is not supported by the current channel realization.

We assume instantaneous and errorless transmissions in the ACK feedback channels. The introduced overhead is outside of the scope of the paper.

\section{A. Source manages the retransmissions}

Two transmission techniques are envisioned based on [4], conventional $D F$ and selective $D F$. The source decides if a new retransmission is required based on the ACK/NACK message from destination after each phase II. Fig. 2-left presents the state diagram when the source manages the ARQ protocol. In such a case, there are two states: $\mathrm{S}_{\mathrm{A}}$, where the transmission is performed from the source and relay and $\mathrm{S}_{\mathrm{C}}$, where destination is able to decode the packet.

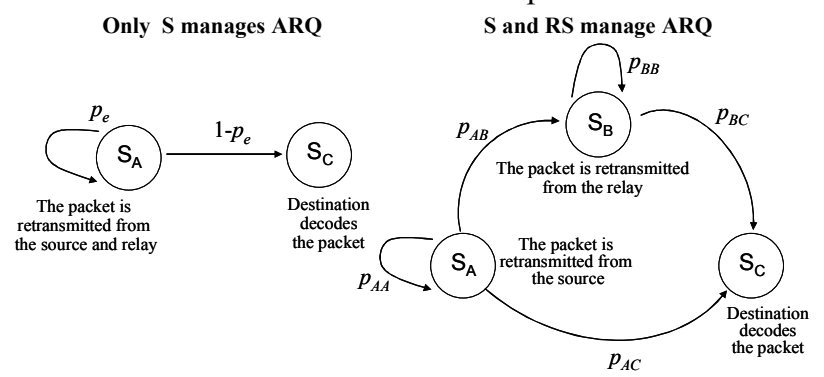

Fig. 2. State diagrams for the ARQ relay-assisted transmission. The ARQ procedure is managed by left) only-source and right) source and relay.

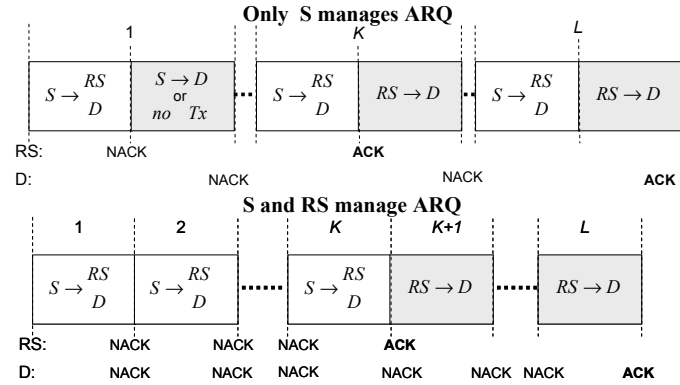

Fig. 3. Example of relay-assisted transmission when: top) only-source and bottom) source and relay manage the ARQ procedure.

An example of the transmission is depicted in Fig. 3-top. The signal received by the relay in phase $\mathrm{I}$ is,

$$
y_{R S}(t)=\frac{1}{\sqrt{L_{1}}} h_{1} x_{s}(t)+w_{r}(t) \text { if } 0 \leq t \leq \alpha T_{s}
$$

where $h_{1}$ and $L_{1}$ are the Rayleigh block-fading coefficient and the pathloss of the source-relay link, respectively, $x_{s}$ is the signal transmitted by the source, $w_{r}$ is the additive white Gaussian noise of power $\sigma_{r}^{2}$ and $\alpha$ stands for the fraction of time allocated to phase I. The instantaneous signal to noise ratio (SNR) is defined by $\gamma_{1}=P\left|h_{1}\right|^{2} / L_{1} / \sigma_{r}^{2}$.

\section{1) Conventional $D F$}

If the relay decodes the received signal, then it transmits $x_{\mathrm{r}}$ in phase II. The signal received at the destination is,

$$
y_{D}(t)= \begin{cases}\frac{1}{\sqrt{L_{0}}} h_{0} x_{s}(t)+w_{d}(t) & \text { if } 0 \leq t \leq \alpha T_{s} \\ \frac{1}{\sqrt{L_{2}}} h_{2} x_{r}\left(t-\alpha T_{s}\right)+w_{d}(t) & \text { if } \alpha T_{s}<t \leq T_{s}\end{cases}
$$

where $h_{0}, L_{0}$ and $\mathrm{h}_{2}, L_{2}$ are the Rayleigh block-fading coefficients and the pathloss of source-destination and relaydestination link, respectively, $w_{d}$ is the white Gaussian noise of power $\sigma_{d}^{2}$. The instantaneous SNR of the $i$ th link is defined by $\gamma_{i}=P\left|h_{i}\right|^{2} / L_{i} / \sigma_{d}^{2}$ with $i=\{0,2\}$. In such a case, the mutual information due to combination of phases is defined by

$$
I_{20}= \begin{cases}\frac{1}{2} \log _{2}\left(1+\gamma_{0}+\gamma_{2}\right) & \text { if } R C \\ \alpha I_{0}+(1-\alpha) I_{2} & \text { if } U C\end{cases}
$$

where $I_{20}$ depends on the transmitting mode of the relay.

If the relay is not able to decode the message, it remains silent. Therefore the mutual information is,

$$
I_{D F}=\left\{\begin{array}{lll}
I_{20} & \text { if } & \alpha I_{1} \geq B \\
\alpha I_{0} & \text { if } & \alpha I_{1}<B
\end{array}\right.
$$

with $B$ a threshold determining if the relay is able to decode.

\section{2) Selective $D F$}

The relay reports the success on decoding the signal received in phase I by sending an ACK/NACK message in the feedback channel. If there is a NACK, the source transmits again in phase II following the same role as the relay ( $\mathrm{RC}$ or UC). In such a case, we assume the change of channel conditions. The signal received at the destination is given by,

$$
y_{D}(t)= \begin{cases}\frac{1}{\sqrt{L_{0}}} h_{0, i} x_{s}(t)+w_{d}(t) & \text { if } 0 \leq t \leq \alpha T_{s} \\ \frac{1}{\sqrt{L_{0}}} h_{0, i+1} x_{s}^{\prime}\left(t-\alpha T_{s}\right)+w_{d}(t) & \text { if } \alpha T_{s}<t \leq T_{s}\end{cases}
$$

where $h_{0, i}$ and $h_{0, i+1}$ stand for the channel coefficient in the source-destination link in the $i$-th and $i+1$-th block 
transmission and $x_{\mathrm{s}}$, denotes the signal transmitted by the source in phase II. The mutual information becomes,

$$
I_{0, S}= \begin{cases}\frac{1}{2} T_{s} \log _{2}\left(1+\gamma_{0, i}+\gamma_{0, i+1}\right) & \text { if } R C \\ \alpha T_{s} \log _{2}\left(1+\gamma_{0, i}\right)+(1-\alpha) T_{s} \log _{2}\left(1+\gamma_{0, i+1}\right) & \text { if } U C\end{cases}
$$

where $\gamma_{0, \mathrm{i}}$ and $\gamma_{0, \mathrm{i}+1}$ are the instantaneous SNR of direct link in different transmission rounds. The mutual information is,

$$
I_{S D F}=\left\{\begin{array}{lll}
I_{20} & \text { if } & \alpha I_{1} \geq B \\
I_{0, S} & \text { if } & \alpha I_{1}<B
\end{array}\right.
$$

\section{B. Source and relay manage the retransmissions}

In this case source and relay collaborate for the retransmission procedure. Both receive the ACK feedback from the destination. Additionally, the source receives the ACK feedback from the relay. In contrast to the previous case, now the phase I is repeated until the message is successfully decoded at the relay (or at the destination, in such a case a new message is transmitted). Afterwards, the relay transmits to destination along multiple phases II until a successfully detection of the message. The state diagram of the relayassisted transmission is presented in Fig. 2-right. There are three states. $S_{A}$ stands for the state where the source is transmitting to the relay and destination (phase I). $S_{B}$ defines the state where the relay has decoded the message and starts transmitting to the destination. Finally, $S_{C}$ denotes the state where the destination has decoded the message. Fig. 3-bottom sketches an example with $K$ phases I and $L-K$ phases II. A new message is transmitted after receiving the ACK from the destination. The signal model defined in (3) and (4) with $\alpha \in[0,1)$ is valid for this protocol. Notice that the destination is reporting an ACK/NACK message after each phase I in order to consider the transition from state $\mathrm{S}_{\mathrm{A}}$ to $\mathrm{S}_{\mathrm{C}}$.

\section{THROUGHPUT EVALUATION}

We have assumed the pure $A R Q$ procedure, [10], which consists in re-transmitting the same signal until the message is successfully received. The link channel conditions vary on each transmission round. The receiver does not consider the signal received in previous time instants. Moreover, the number of re-transmissions can be infinite. Through this section we will assume the following definitions,

$$
\begin{array}{ll}
p_{0}(\alpha, B)=\operatorname{Pr}\left(\alpha I_{0}<B\right) & p_{1}(\alpha, B)=\operatorname{Pr}\left(\alpha I_{1}<B\right) \\
p_{20}(\alpha, B)=\operatorname{Pr}\left(I_{20}<B\right) & p_{0, S}(\alpha, B)=\operatorname{Pr}\left(I_{0, S}<B\right)
\end{array}
$$

where $p_{0}, p_{1}$ and $p_{20}$, which depend on $\alpha$ and $B$, stand for the outage probability of the source-destination, source-relay and joint transmission from the source and relay to destination, respectively. The variable $p_{0, \mathrm{~S}}$ is the outage probability due to the transmission from the source in two phases, (8). The exact equations under RC or UC are derived in the Appendix by equations (23) and (24). Those equations are also valid for $p_{0, \mathrm{~S}}$ using $\rho_{2}=\rho_{0}$. Moreover, $p_{0}, p_{1}$ are defined by (22).

\section{A. Source manages the retransmissions}

When the source controls the retransmission procedure the throughput of the system with pure ARQ protocol is given by,

$$
\mathcal{T}=\max _{B, \alpha} \frac{B}{E\{t\}}=\max _{B, \alpha} \frac{B}{T_{s}}\left(1-p_{e}\right)
$$

where $\mathrm{E}\{t\}$ denotes the average time needed for successfully receiving the $B$ bits and $p_{e}$ is the outage probability of the transmission. The value $1-p_{e}$ describes the transition probability from $\mathrm{S}_{\mathrm{A}}$ to $\mathrm{S}_{\mathrm{C}}$ in Fig. 2-left. For the relay-assisted transmission, $p_{e}$ depends on $B$ and the fraction of time allocated to each phase, $\alpha$. According to the mutual information for the conventional DF shown in (6), the outage probability of the transmission is,

$$
p_{e}^{D F}=p_{20}(\alpha, B)\left(1-p_{1}(\alpha, B)\right)+p_{1}(\alpha, B) p_{0}(\alpha, B)
$$

where $p_{20}, p_{1}$ and $p_{0}$ are defined in (10). On the other hand, the outage probability for the selective DF, (9) is given by,

$$
p_{e}^{S D F}=p_{20}(\alpha, B)\left(1-p_{1}(\alpha, B)\right)+p_{1}(\alpha, B) p_{0, S}(1, B)
$$

with $p_{0, \mathrm{~S}}$ defined in (10).

\section{B. Source and relay manage the retransmissions}

Since the main objective of the relay-assisted transmission is to combine the signals received through different phases, in this scheme the destination must store the signal received in the phase I where the relay successfully decode the message ( $K$-th phase I in Fig. 3-bottom). Afterwards, the destination will try to decode the message considering that stored signal and the signal received by the last transmission from the relay (phase II). We can increase the complexity by allowing to the destination storing the phase I with the maximum SNR or allowing to the relay and destination chase combining all the received signals. This second case is investigated in [14].

The transition probabilities depicted in Fig. 2-right are,

$$
\begin{array}{ll}
p_{A B}=\operatorname{Pr}\left(\alpha T_{s} \cdot I_{1}>B\right) \operatorname{Pr}\left(\alpha T \cdot I_{0}<B\right)=\left(1-p_{1}\right) p_{0} \\
p_{A C}=\operatorname{Pr}\left(\alpha T_{s} \cdot I_{0}>B\right)=1-p_{0} & p_{A A}=1-p_{A B}-p_{A C} \\
p_{B B}=\operatorname{Pr}\left(T_{s} \cdot I_{20}<B \mid \alpha T_{s} \cdot I_{0}<B\right) & p_{B C}=1-p_{B B}
\end{array}
$$

where the probabilities are derived in the Appendix, $p_{0}, p_{1}$ by (22) and $p_{B B}$ under RC or UC in (28) and (29), respectively.

In order to evaluate the average time devoted for transmitting a packet we will consider two types of events: $E_{0}$ and $E_{k}$ for $k=1 . . \infty$. The event $E_{0}$ denotes those situations where the destination has been able to decode the packet using only phase I (transitions $S_{\mathrm{A}}-S_{\mathrm{C}}$ ). The average time associated for this event is given by,

$$
E\left\{t_{E_{0}}\right\}=\alpha T_{s} p_{A C} \sum_{n=1}^{\infty} n p_{A A}^{n-1}=\alpha T_{s} \frac{p_{A C}}{\left(1-p_{A A}\right)^{2}}
$$

On the other hand, the event $E_{k}$ describes the transitions $S_{\mathrm{A}}-S_{\mathrm{B}^{-}}$ $S_{\mathrm{C}}$ when the relay has decoded the message after the $k$-th phase I. The average time associated to this event becomes,

$$
\begin{gathered}
E\left\{t_{E_{k}}\right\}=p_{A A}^{k-1} p_{A B} p_{B C} \sum_{n=1}^{\infty}\left(k \alpha T_{s}+n(1-\alpha) T_{s}\right) p_{B B}^{n-1}= \\
=p_{A A}^{k-1} p_{A B}\left(1-p_{B B}\right)\left[\frac{k \alpha T_{s}}{\left(1-p_{B B}\right)}+\frac{(1-\alpha) T_{s}}{\left(1-p_{B B}\right)^{2}}\right]
\end{gathered}
$$

Finally the expected time for delivering a packet is given by, 


$$
E\left\{t_{A R Q}\right\}=\sum_{k=0}^{\infty} E\left\{t_{E_{k}}\right\}=\frac{\alpha T_{s}}{\left(1-p_{A A}\right)^{2}} p_{A B}+\frac{(1-\alpha) T_{s}}{p_{B C}\left(1-p_{A A}\right)} p_{A B}
$$

Now, the optimal throughput of the system is attained by,

$$
\mathcal{T}_{A R Q}=\max _{\alpha, B} \frac{B}{E\left\{t_{A R Q}\right\}}=\max _{\alpha, B} \frac{B}{T_{s}}\left(\frac{\alpha}{1-p_{A A}}+\frac{(1-\alpha) p_{A B}}{\left(1-p_{A A}\right) p_{B C}}\right)^{-1}
$$

Therefore by optimizing the time allocated for phase I $(\alpha)$ and the selected rate $(B)$ we maximize the throughput.

\section{RESULTS}

The relay-assisted transmission is evaluated in a simple scenario where the source-destination distance is normalized to one. The RS is placed at distance $d$ from the source and (1-d) from the destination. The pathloss of each link $\left(L_{\mathrm{i}}\right)$ is proportional to the square of the distance between the terminals (propagation exponent equal to 2). The channel coefficients are Rayleigh distributed and the average SNR of each link is defined by (2). We have assumed that the noise power at the destination and relay is the same. By defining the average SNR in the source-destination link, $\rho_{0}\left(L_{0}=1\right)$, we also define the average SNR of the other links, $\rho_{1}$ and $\rho_{2}$.

Fig. 4 presents the optimized throughput for the schemes analyzed in section III as a function of the position of the relay when $\rho_{0}=0 \mathrm{~dB}$. When the source controls the retransmission procedure, conventional DF operates under RC or UC (DFRC-S or DF-UC-S) and the selective $D F$ only considers UC (S-DF-S). On the other hand, when source and relay manage the retransmissions, we identify the different transmission schemes by DF-UC-SR or DF-RC-SR. The best throughput is obtained for the DF-UC-SR. Notice that DF-RC-S presents lower throughput than the direct transmission when the relay is placed far from the source. Moreover, DF-RC-SR can even outperform the S-DF-S when the relay is close to the source.

Another important aspect to be investigated is the position of the relay where the maximum throughput is reached for the different strategies. To this end, Fig. 5 explores the additive throughput gain, $\Delta \mathcal{T}$, for the DF-UC-S, S-DF-S and DF-UC-SR when the relay is placed at $d=\{0.1,0.5,0.9\}$ as a function of $\rho_{0}$. When only the source manages the retransmission, the best positions for the relay are close to the destination ( $d=0.9$ ), although for $\rho_{0} \geq 12 \mathrm{~dB}$ the DF-UC-S gets a better throughput if the relay is placed at $d=0.5$. In contrast, when source and relay control the retransmissions (DF-UC$\mathrm{SR})$ the best positions of the relay are at middle distance and close to the source ( $d=0.5$ if $\rho_{0} \leq 12 \mathrm{~dB}$ and $d=0.1$ if $\left.\rho_{0}>12 \mathrm{~dB}\right)$. Notice the significant throughput gain obtained when source and relay manage the retransmission procedure.

\section{CONCLUSIONS}

In this paper we have analyzed the relay-assisted transmission in combination with the pure- $A R Q$ protocol, analyzing the cases where only-source or source and relay manage the retransmission procedure, providing closed-form expressions for the optimal throughput. Results have shown that the optimal position of the relay depends on which terminals manage the ARQ. The best throughput gains are attained when source and relay manage the retransmissions and the relay is closer to the source than to the destination, which is consistent with the behavior of the achievable rate of DF relay schemes.

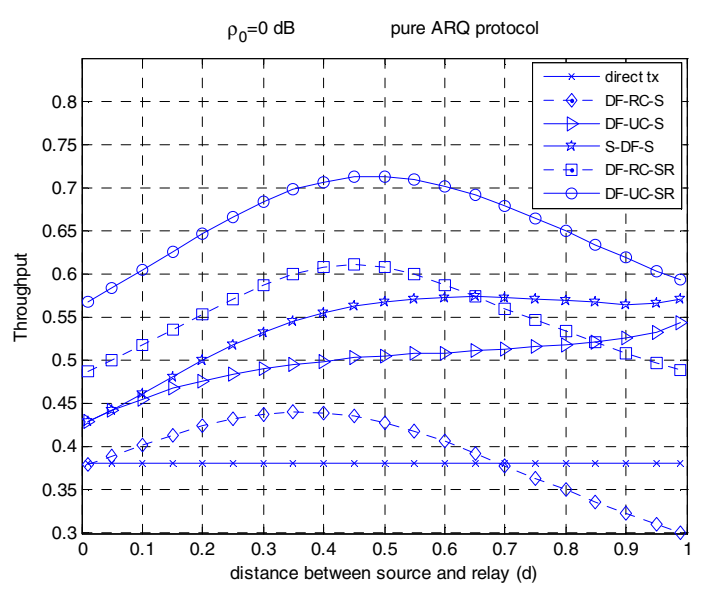

Fig. 4. Throughput as a function of the distance between the source and relay. $\rho_{0}=0 \mathrm{~dB}$. The source manages the retransmission procedure (direct tx, DF-RC$\mathrm{S}, \mathrm{DF}-\mathrm{UC}-\mathrm{S}$ and S-DF-S) and source and relay manage the retransmissions (DF-RC-SR, DF-UC-SR)

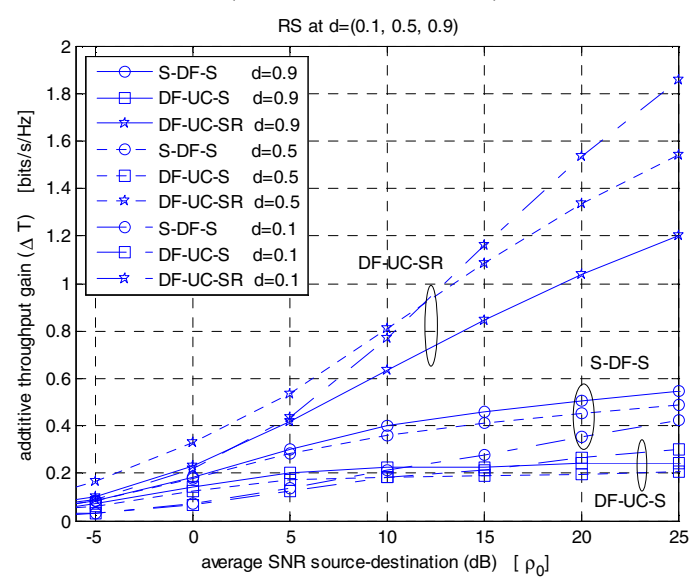

Fig. 5. Additive throughput gain of the relay-assisted transmission as a function of the average $\operatorname{SNR}\left(\rho_{0}\right)$. The relay works under UC. The source manages the retransmission procedure (DF-S, DF-UC-S and S-DF-S) and source and relay manage the retransmissions (DF-RC-SR, DF-UC-SR).

\section{APPENDIX}

This appendix unearths the outage probability equations defined in section III.A and III.B when instantaneous SNR, $\gamma_{i}$, follows an exponential distribution with the mean $\rho_{i}$ defined in (2). The relay transmission based on RC or UC will be considered to derive the expressions for $p_{20},(10)$, and $p_{B B}$, (14) , when only-source or source and relay control the ARQ procedure. The probability density function $(p d f)$ of $\gamma_{i}$ and the mutual information $\beta I_{i}$, defined in [7],[13] are given by,

$$
f_{\gamma_{i}}(z)=\frac{1}{\rho_{i}} \exp \left(\frac{-z}{\rho_{i}}\right), f_{\beta I_{i}}(z)=\frac{\ln (2)}{\beta \rho_{i}} \exp \left(\frac{-\varphi(z / \beta)}{\rho_{i}}\right) 2^{\frac{z}{\beta}}
$$

where both equations are only defined for $z \geq 0$ and

$$
\varphi(y)=2^{y}-1
$$

For sake of notation simplicity in the equations to be 
presented in this section we will define,

$$
\lambda=\frac{B}{T_{s}}, \quad \omega\left(\rho_{i}, y\right)=1-\exp \left(-\frac{\varphi(y)}{\rho_{i}}\right), \quad \varepsilon_{0}=\varphi(2 \lambda)
$$

\section{A. Source-relay and source-destination links}

The outage probability in the source-destination $\left(p_{0}\right)$ and source-relay $\left(p_{1}\right)$ is given by,

$$
p_{i}(\alpha, B)=\omega\left(\rho_{i}, \lambda / \alpha\right) \quad i=0,1
$$

\section{B. Source manages the retransmissions}

In the following the $p_{20}$ defined in (10) will be derived.

\section{1) Repetition coding $(R C)$}

Under this mode the destination combines the signals received from the source and relay under chase combining. In a nutshell, we have a sum of exponential random $\left(\gamma_{0}\right.$ and $\left.\gamma_{2}\right)$ variables [13] and the outage probability is defined by,

$$
\begin{aligned}
& p_{20}^{R C}=\operatorname{Pr}\left(\frac{1}{2} T_{s} \log _{2}\left(1+\gamma_{0}+\gamma_{2}\right)<B\right)=\operatorname{Pr}\left(\gamma_{0}+\gamma_{2}<\varepsilon_{0}\right)= \\
& = \begin{cases}1-\exp \left(\frac{-\varepsilon_{0}}{\rho_{0}}\right)\left(1+\frac{\varepsilon_{0}}{\rho_{0}}\right) & \text { if } \rho_{2}=\rho_{0} \\
1-\frac{1}{\rho_{2}-\rho_{0}}\left(\rho_{2} \exp \left(\frac{-\varepsilon_{0}}{\rho_{2}}\right)-\rho_{0} \exp \left(\frac{-\varepsilon_{0}}{\rho_{0}}\right)\right) & \text { if } \rho_{2} \neq \rho_{0}\end{cases}
\end{aligned}
$$

where $\rho_{0}, \rho_{2}$ stand for the average SNR and $\varepsilon_{0}$ is given in (21).

\section{2) Unconstrained coding (UC)}

We provide an integral equation to be evaluated numerically,

$$
\begin{aligned}
& p_{20}^{U C}=\operatorname{Pr}\left(\alpha T_{s} \cdot I_{0}+(1-\alpha) T_{s} \cdot I_{2}<B\right)=\int_{0}^{\lambda} \int_{0}^{\lambda-t} \frac{\ln (2)}{(1-\alpha) \rho_{2}} \\
& \cdot \exp \left(-\frac{\varphi(z /(1-\alpha))}{\rho_{2}}-\frac{\varphi(t / \alpha)}{\rho_{0}}\right) 2^{\frac{z}{1-\alpha}} \frac{\ln (2)}{\alpha \rho_{0}} 2^{\frac{t}{\alpha}} d z d t \\
&=\omega\left(\rho_{0}, \lambda / \alpha\right)-\kappa\left(\alpha, B, T_{s}, \rho_{0}, \rho_{2}\right)
\end{aligned}
$$

where $\kappa$ depends on the variables $\alpha, B, T_{s}, \rho_{0}$ and $\rho_{2}$,

$$
\kappa=\frac{\ln (2)}{\alpha \rho_{0}} \int_{0}^{\lambda} 2^{\frac{z}{\alpha}} \exp \left(-\frac{\varphi(z / \alpha)}{\rho_{0}}-\frac{\varphi((\lambda-z) /(1-\alpha))}{\rho_{2}}\right) d z
$$

\section{Proposed relay-assisted transmission}

The derivation of $p_{B B}$, defined in (14), under RC or $\mathrm{UC}$ will be presented. We follow similar guidelines as in the previous sub-section, but now conditioning on the event that the destination was not able to successfully decode the message in phase I, because we are on state $S_{B}$ in Fig. 2-right.

\section{1) Repetition coding $(R C)$}

$$
\begin{gathered}
p_{B B}^{R C}=\operatorname{Pr}\left(\frac{1}{2} T_{s} \log _{2}\left(1+\gamma_{0}+\gamma_{2}\right) \leq B \mid \frac{1}{2} T_{s} I_{0}<B\right)= \\
=\operatorname{Pr}\left(\gamma_{0}+\gamma_{2} \leq \varepsilon_{0} \mid \gamma_{0}<\varepsilon_{0}\right)=\int_{0}^{\varepsilon_{0}} \operatorname{Pr}\left(\gamma_{2} \leq \varepsilon_{0}-t\right) f_{\gamma_{0} \mid \gamma_{0}<\varepsilon_{0}}(t) d t(26)
\end{gathered}
$$

where the $p d f$ of the variable $\gamma_{0}$ conditioned to $\gamma_{0} \leq \varepsilon_{0}$ with $\varepsilon_{0}$ defined in (21) is given by,

$$
f_{\gamma_{0} \mid \gamma_{0}<\varepsilon_{0}}(z)=\frac{1}{\omega\left(\rho_{0}, \varepsilon_{0}\right) \rho_{0}} \exp \left(\frac{-z}{\rho_{0}}\right) \quad 0 \leq z \leq \varepsilon_{0}
$$

Under those circumstances the outage probability becomes,

$$
\begin{aligned}
p_{B B}^{R C}= & \operatorname{Pr}\left(\gamma_{0}+\gamma_{2}<\varepsilon_{0} \mid \gamma_{0}<\varepsilon_{0}\right)= \\
= & \text { if } \rho_{0}=\rho_{2} \\
1+\frac{\varepsilon_{0}}{\omega\left(\rho_{0}, \varepsilon_{0}\right) \rho_{0}} & \text { if } \rho_{0} \neq \rho_{2} \\
1+\frac{\rho_{2}\left(1-\exp \left(\varepsilon_{0} / \rho_{0}-\varepsilon_{0} / \rho_{2}\right)\right)}{\left(\rho_{0}-\rho_{2}\right) \omega\left(\rho_{0}, \varepsilon_{0}\right)} &
\end{aligned}
$$

2) Unconstrained coding (UC)

$$
\begin{gathered}
p_{B B}^{U C}=\operatorname{Pr}\left(T_{s} \cdot I_{20}<B \mid \alpha T_{s} \cdot I_{0}<B\right)=\int_{0}^{\lambda} \int_{0}^{\lambda-t} \frac{\ln (2)}{(1-\alpha) \rho_{2}} \\
\cdot \exp \left(-\frac{\varphi(z /(1-\alpha))}{\rho_{2}}-\frac{\varphi(t / \alpha)}{\rho_{0}}\right) \frac{2^{\frac{z}{1-\alpha}} \ln (2) 2^{\frac{t}{\alpha}}}{\omega\left(\rho_{0}, \lambda / \alpha\right) \alpha \rho_{0}} d z d t \\
=1-\kappa\left(\alpha, B, T_{s}, \rho_{0}, \rho_{2}\right) \omega\left(\rho_{0}, \lambda / \alpha\right)^{-1}
\end{gathered}
$$

where $\omega$ and $\kappa$ are defined in (21) and (25), respectively. In the previous equation it has been assumed that $f_{\alpha I_{0} \mid \alpha I_{0}<B}=f_{\alpha I_{0}} \omega\left(\rho_{0}, \lambda / \alpha\right)^{-1}$ and $f_{\alpha I_{0}}$ given in (19).

\section{REFERENCES}

[1] E.C. van der Meulen, "Three-terminal communication channels", Adv. Appl. Prob., vol.3, pp. 120-154, 1971

[2] T.M.Cover, A.El Gamal, "Capacity theorems for the relay channel", IEEE Trans. on Information Theory, vol.25,no.5,pp.1468-1489,Sep.1979

[3] A.Sendonaris, E.Erkip, B.Aazhang, "User cooperation diversity-part I: System description", IEEE Trans. on Communications, vol.51, no.11, pp. 1927-1938, Nov. 2003.

[4] J.N.Laneman, D.N.C.Tse, G.W.Wornell, "Cooperative diversity in wireless networks: Efficient protocols and outage behavior", IEEE Trans. Information Theory, vol. 50, no.12, pp.3062-3080, Dec. 2004.

[5] G.Kramer, M.Gastpar, P.Gupta, "Cooperative strategies and capacity theorems for relay networks", IEEE Trans. on Information Theory, vol. 51, no. 9, Sep. 2005.

[6] E.Stauffer, Ö.Oyman, R.Narasimhan, A.Paulraj, "Finite-SNR diversitymultiplexing tradeoffs in fading relay channels", IEEE Journal on Selected Areas in Communications, vol. 25, no.2, pp.245-257, Feb.2007.

[7] M.N. Khormuji, E.G.Larsson, "Analytical results on block length optimization for decode-and-forward relaying with CSI feedback", in Proc. $8^{\text {th }}$ IEEE Workshop on Signal Processing Advances in Wireless Communications (SPAWC-2007), June 2007, Helsinki, Finland.

[8] D.P.Palomar, A.Agustin, O.Muñoz, J.Vidal, "Decode and forward protocol for cooperative diversity in multi-antenna wireless networks", in Proc. IEEE Annual Conference on Information Sciences and Systems (CISS-2004), Princeton, NJ, USA, Feb. 2004.

[9] Z.Lin, E.Erkip, M.Ghosh, "Adaptive modulation for coded cooperative systems", in Proc. $6^{\text {th }}$ IEEE Workshop on Signal Processing Advances in Wireless Communications (SPAWC-2005), June 2005, New York, USA.

[10] D.J.Costello, J.Hagenauer, H.Imai, S.B.Wicker, "Applications of errorcontrol coding”, IEEE Trans. IT, vol.44, no.6, pp.2531-2560, Oct. 1998.

[11] B.Zhao, M.C.Valenti, "Practical Relay Networks: A Generalization of Hybrid-ARQ", IEEE Journal on Selected Areas in Communications, vol. 23, no.1, pp.7-18, Jan.2005

[12] T.Tabet, S.Dusad, R.Knopp, "Diversity-Multiplexing-delay tradeoff in half-duplex ARQ relay channels", IEEE Trans. on Information Theory, vol.53, no.10, Oct. 2007

[13] M.K.Simon, Probability Distributions Involving Gaussian Random Variables: A Handbook for Engineers and Scientists. Springer 2002

[14] A.Agustin, J.Vidal, O.Muñoz, "Analytical resource optimization for the DF relay-assisted transmission under HARQ”, in Proc. IEEE Vehicular Technology Conf (VTC-Spring), April 2009, Barcelona, Spain 